\title{
Immunohistochemical Study of OTUB1 Expression in Colon Cancer
}

\section{Limei Lian ${ }^{1}$, Xue-chun Wang ${ }^{2}$, Wei-Na Jiang ${ }^{3}$, Hua Chen C4}

\author{
${ }^{1}$ Department of Pathology, Qingdao University Medical College, Qingdao, China \\ ${ }^{2}$ Department of Pathology, Qingdao Municipal Hospital, Qingdao, China \\ ${ }^{3}$ Department of Radiology, Qingdao Municipal Hospital, Qingdao, China \\ ${ }^{4}$ Department of Pathology, Qingdao Municipal Hospital, No. 5 Donghai Middle Rd., 266071 Qingdao, China
}

\begin{abstract}
Background: OTUB1 is a new member of ovarian-tumor-domain-containing proteases (OTUs), a deubiquitinating enzymes family (DUBs), which is a cysteine proteasome-associated DUB to be involved in the Ub-dependent degradation of proteins. Therefore the expression of OTUB1 in colon cancer was examined and compared with precancerous lesion and normal colonic mucosa.
\end{abstract}

Material/Methods: Tissue samples were obtained from 60 patients, and we took a piece of tissue from three different parts of the same patient, colon cancer, precancerous lesion and normal mucosa, respectively. Immunohistochemistry were carried out in colon cancer, precancerous lesion and normal mucosa.

Results: Precancerous lesions and colon cancer tissues showed strong expression of OTUB1 in the cell cytoplasm. The expression of OTUB1 protein in the colon cancer was significantly higher than precancerous lesion and normal mucosa $(\mathrm{P}<0.05)$. Moreover, its expression in normal mucosa, precancerous lesion and colon cancer showed a gradually ascendant trend $(\mathrm{P}<0.05)$.

Conclusion: The protein level of OTUB1 makes a greater contribution to colon cancer development.

Keywords: OTUB1; Colon cancer; Immunohistochemistry

\section{Introduction}

It is generally accepted that approximately $95 \%$ of colorectal cancer arise from adenomatous polyps, which are monoclonal derivatives of a mutated epithelial stem cell [1]. Colorectal cancer is one of the commonest malignant tumors, which is the third most commonly diagnosed cancer in males and the second in females, with an estimated 1.4 million cases and 693,900 deaths occurring in 2012 [2], and what is more, the disease-specific mortality rate is nearly $33 \%$ even in the developed countries [3], In the past 30 years, many countries, including China, colon cancer incidence showed an increasing trend[4]. For the treatment of colon cancer is still based on surgery, radiotherapy and chemotherapy supplemented treatments, such as much as possible to kill tumor cells, to reduce the number of tumor cells to achieve the therapeutic effect. However, colon cancer recurrence of postoperative 5-year is remain with low survival rate, high transfer rate and resistance to high [3] . Therefore, exploring the pathogenesis of colon cancer, has an important significance in the prevention, earlier diagnosis and target therapy of colon cancer.

One family of the most recently recognized deubiquitinating enzymes (DUBs) were the ovariantumor-domain-containing proteases (OTUs), which were of considerable interest to us due to its conserved sequences in viruses, bacteria, plants, yeast and humans, and they were involved in immunity and inflammation[5]. OTU domain-containing ubiquitin aldehyde-binding protein 1 (OTUB1), one member of OTUs, is ubiquitously expressed in human tissues (e.g. kidney, intestine, brain, liver, and lung) [6,7]. During the proteolysis by proteasome, the removal of the ubiquitin chain from the substrate by proteasomeassociated DUBs such as OTUB1 is a key to allow the

This article is published under the terms of the Creative Commons Attribution License 4.0

Author(s) retain the copyright of this article. Publication rights with Alkhaer Publications.

Published at: http://www.ijsciences.com/pub/issue/2016-02/

DOI: 10.18483/ijSci.906; Online ISSN: 2305-3925; Print ISSN: 2410-4477 
passage of the unfolded polypeptide through a narrow constriction into the proteolytic chamber of the proteasome core particle, where proteolysis ensues $[8,9]$.

Sun et al. found that OTUB1 could induce the p53dependent cellular apoptosis and inhibit proliferation by regulating the ubiquitination of p53[10]. Herhaus et al. found that Otub1 enhanced TGF-mediated gene transcription, through bonding with phosphorylation of SMAD2/3 and rescuing phospho-SMAD2/3 from proteasomal degradation[11]. Therefore, the effect of OTUB 1 on the biological behavior of tumor cells need to be further analyzed. In this study, we carried out immunohistochemistry to investigate the expression of OTUB1 in normal colonic mucosa, dysplasia lesions and colon cancer to evaluate the OTUB1 expression.

\section{Materials and methods Tissue samples}

All tissues used in this study were obtained from 60 patients with surgically treated for colon cancer in Qingdao Municipal Hospital from May 2010 to April 2015. At the time of surgery, all patients were not subjected to chemotherapy and radiotherapy. We collected normal mucosa tissues $(5 \mathrm{~cm}$ away from the tumor edge), precancerous lesion and carcinomas of the colon from the same patient. Sixty precancerous lesion samples were classified as 27 adenoma (independent existence near the colon cancer ) and 33 adenomatous hyperplasia (in the edge of colon cancer). The institutional review board approved this study. All samples were formalin-fixed and paraffin-embedded (FFPE) and underwent immunohistochemistry staining.

\section{Reagents and antibodies}

Rabbit anti-OTUB1 polyclonal antibody (Cat No. ab103995) was purchased from Abcam (Hong Kong, China). Polymer enhancer (Reagent A), polymerized horseradish peroxidase-anti-mouse/rabbit IgG (ReagentB), citrate buffer $\quad(\mathrm{pH}=6.0)$, diaminobenzidine (DAB), phosphate buffer saline (PBS) were purchased from Maixin (Fuzhou, China).

\section{Immunohistochemistry}

Immunohistochemistry staining was carried out following the manufacturer's protocols. Paraffin sections (4 $\mu \mathrm{m}$ thick) were deparaffined in two changes of xylene for 5 minutes each and then hydrated in decreasing concentrations of ethanol (100, 95, 95, and 75\%) and rinsed in water. After that, all sections were incubated with $3 \%$ hydrogen peroxide for $15 \mathrm{~min}$ to block endogenous peroxidase activity at first. Antigen retrieval was routinely performed by incubation in sodium citrate buffer $(\mathrm{pH}$ 6.0) and heated in high pressure method for 3 minutes. The primary antibody (1:3200) were incubated for $90 \mathrm{~min}$ at room temperature. After rinsing, sections was performed with Reagent A and Reagent B subsequently for $15 \mathrm{~min}$. Diaminobenzidine (DAB) was used as the chromogen substrate. The sections were then counterstained with hematoxylin. Primary antibody is replaced with PBS as a negative control group. The human brain tissue was used as positive control according to the manufacture's instructions. Using a light microscope to analyze immunostaining of OTUB1 and taking digital pictures from random areas of slides.

The following criteria were considered to evaluate the immunoreactivity score (IRS): (a) staining intensity: no immunoreactivity was scored as 0 , weak immunostaining was 1 , moderate immunostaining was 2 , and strong immunostaining was 3; (b) percentage of positive tumor cells: $0-5 \%$ was $0,6-25 \%$ was $1,26-$ $75 \%$ was 2 , and $75-100 \%$ was 3 . IRS was calculated by the multiply of the above two values. The final score of $0-3$ was categorized as low expression, and 4-9 was categorized as high expression.

\section{Statistical Analysis}

The Chi-square test was applied to compare the differences in each group regarding OTUB1 expression. The level of statistical significance was defined as $\mathrm{p}<0.05$. Statistical analysis were conducted using SPSS 17.0 statistical software (SPSS, Chicago, IL, USA)

Results

Table1:OTUB1 expression in normal mucosa, adenoma polyp and adenomatous hyperplasia

\begin{tabular}{|c|c|c|c|c|}
\hline & \multirow[b]{2}{*}{$\mathrm{n}$} & \multicolumn{2}{|c|}{ OTUB 1} & \multirow[b]{2}{*}{ P-value } \\
\hline & & Low expression $\mathrm{n}(\%)$ & High expression $\mathrm{n}(\%)$ & \\
\hline Normal mucosa & 60 & $49(81.67)$ & $11(18.33)$ & \\
\hline Adenoma polyp & 27 & $12(44.44)$ & $15(55.56)$ & $<0.05 *$ \\
\hline Adenomatous hyperplasia & 33 & $16(48.48)$ & $17(51.52)$ & $<0.05^{*}>0.05 * *$ \\
\hline
\end{tabular}

$\mathrm{p}$ - determined by the Chi-square test; $*_{-}$in relation to the normal mucosa; $*^{*}$ - in relation to adenoma polyp 
Table2:OTUB1 expression in normal mucosa, precancerous lesion and adenomatous colon cancer.

\begin{tabular}{|c|c|c|c|c|}
\hline & \multirow[b]{2}{*}{$\mathrm{n}$} & \multicolumn{2}{|r|}{ OTUB1 } & \multirow[b]{2}{*}{ P-value } \\
\hline & & Low expression $\mathrm{n}(\%)$ & High expression $\mathrm{n}(\%)$ & \\
\hline Normal mucosa & 60 & $49(81.67)$ & $11(18.33)$ & \\
\hline Precancerous lesion & 60 & $28(46.67)$ & $32(53.33)$ & $<0.05$ \\
\hline Colon cancer & 60 & $17(28.33)$ & $43(71.67)$ & \\
\hline
\end{tabular}

$\mathrm{p}$ - determined by the Chi-square test

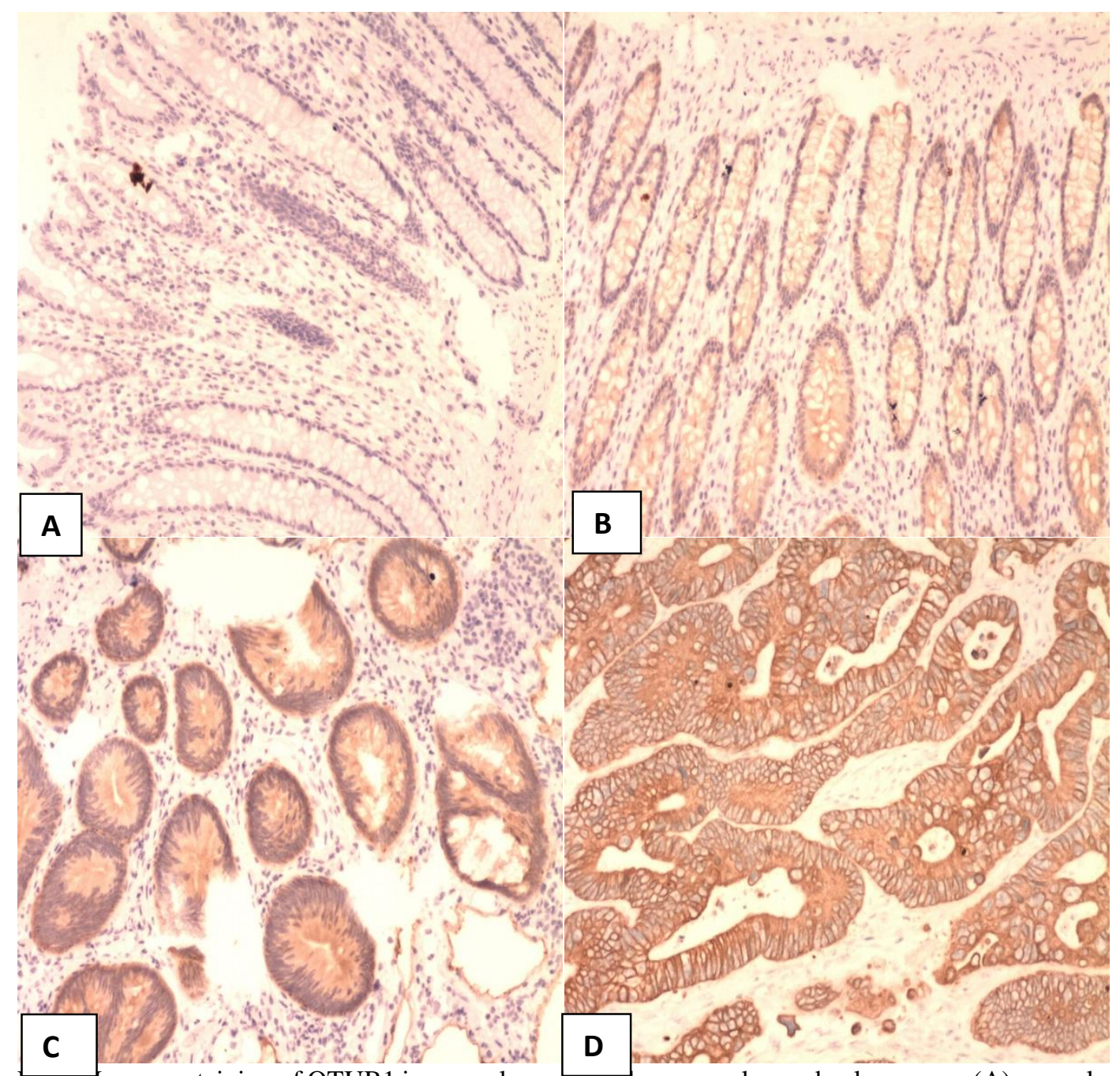

Fig. 1. Immunostaining of OTUB1 in normal mucosa, adenoma polyp and colon cancer.(A) normal colon mucosa was negative for OTUB1;(B) Moderate staining reaction in normal mucosa; (C) Strong immunoreactivity in adenoma polyp;(D) ) Colon cancer was positive for OTUB1.

\section{OTUB1 Expression Showed Low Protein Level in Normal Mucosa.}

The positive anti-OTUB1 immunostaining was found mainly in the cytoplasm. Low OTUB1 expression (49 cases) was observed in normal colon mucosa, only 11 cases showed high OTUB1 expression (Table 1). There was no immunostaining in majority of the normal mucosa (Figure 1A), and even in samples of these with high OTUB1 expression, the staining intensity was moderate ( Figure 1B).

\begin{abstract}
The Immunoreactivity of Adenoma was Closed to Adenomatous Hyperplasia.

High expression of OTUB1 in adenoma and dysplasia lesions were $55.56 \%$ and $51.52 \%$, respectively (Table $1)$. And the expression of OTUB1 in adenoma and precancerous lesion was not significantly different $(\mathrm{P}>0.05)$. We infered that the high level of OTUB1 expression in adenoma was closed to adenomatous hyperplasia. Therefore, we calculated the OTUB1 expression level in 27 adenoma and 33 adenomatous hyperplasia together. Approximately $53.33 \%$ of precancerous lesion showed strong OTUB1
\end{abstract}


immunoreactivity (Figure 1C). About $46.67 \%$ of precancerous lesion expressed low protein levels. The results of the chi-squared test $(\mathrm{P}<0.05)$ showed a significant difference between precancerous lesion and normal mucosa.

\section{Colon Cancer Had High OTUB1 Expression.}

Approximately $71.67 \%$ of colon cancerous tissues were strongly positive for OTUB1-antibody (Figure 1D). The stroma of colon tissues were totally unreactive. Statistically significant difference was found between colon cancer and normal mucosa. Furthermore, there were significant differences in colon cancer that compared with precancerous lesion. Moreover, the results of the chi-squared test showed an increasing trend in normal mucosa, adenoma, carcinoma of the colon $\quad(\mathrm{P}<0.05)$. The results of anti-OTUB1 immunostaining of normal mucosa, precancerous lesion and colon cancer are presented in table 2 .

\section{Discussion}

The OTU Characterized by the presence of ovarian tumor domaincontaining sequences acts as general deubiquitinases. OTU1 and OTU2 were first confirmed to have deubiquitinase activity in vitro. otub1 gene is located at chromosomal position 11q13.1, which has an OTU domain of 130 amino acids, and is ubiquitously expressed in human tissues [12]. In this study, OTUB1 immunoreactivity appeared in the cytoplasm immediately away from the tumor, but its intensity was weak and only locally moderate. It was evidently decreased compared with colon cancer. In contrast, high OTUB1 deposition was found in both cases of adenoma and carcinoma of the colon.

The Ubiquitin-proteasome pathway is play an important role in regulating intracellular protein metabolism, involving cell cycle, signal transduction, DNA repair, receptor function, development, differentiation, inflammatory reaction and many other aspects[13]. OTUs are part of the DUBs family, which play important roles in mediating the processes of proteins ubiquitination and degradation[14]. DUBs can hydrolyze isopeptide bonds between ubiquitin and folded proteins, remove the ubiquitin or poly ubiquitins from target proteins, and interfere the degradation of substrates in the Ub-dependent pathway[15].

OTUB1 has emerged as a unique DUB that has many ways to influence Ubiquitin-proteasome pathway. Recently studies in primate species, including the human, suggest that OTUB1 regulations closely associated with EGFR and EGF-like molecules via paracrine signaling[16,17]. Zhong et al. found that OTUB 1 acts as a deubiquitination via activation of the $\beta$-catenin / TCF deubiquitinating activity[18].
Recently, OTUB1 reg ulates p53 through noncanonical suppression of the ubiquitin-conjugating enzyme (E2) activity of UbcH5 (also called UbE2D), leading to the inhibition of MDM2- mediated p53 ubiquitination[19]. In this sense, OTUB1 may promote colon cancer proliferation and metastasis through this signal pathway. OTUB1 overexpression was $18.33 \%, 53.33 \%$, and $71.67 \%$ in normal mucosa, adenoma and colon cancer tissues, respectively. we obtained that the level of OTUB1 expression in carcinoma of the colon was higher than other colon tissues.

In conclusion, the present study demonstrated that an increasing trend in normal mucosa- precancerous lesion tissues - carcinoma of the colon. The result indicated OTUB1 may be responsible for the development of colon cancer. However, the mechanisms through which OTUB1 works are still unknown. Therefore, further investigation is necessary for better understanding the detail mechanisms, which can provide new strategy and new target for colon cancer treatment.

\section{Acknowledgement}

This work was supported by the grants from Science \& Technology Development Foundation of Qingdao City (No. 12-1-4-16-[3]-jch; 08-2-1-4-nsh).

\section{References}

1. Bond JH. Polyp guideline: diagnosis, treatment and surveillance for patients with colorectal polyps[ J ]. Am J Gastroenterol, 2000, 95(11): 3053-63.

2. Lindsey A.Torre, Freddie Bray, et al. Global Cancer Statistics 2012[ J ]. CA CANCER J CLIN 2015; 65:87-108.

3. D. Cunningham, W. Atkin, H. Lenz, H.T. Lynch, B. Minsky, B. Nordlinger, N.Starling. Colorectal cancer[ J ]. Lancet 375 (2010) : 1030-1047.

4. Shaukat A, Mongin SJ, Geisser MS, et al ,Long-term mortality after screening for colorectal cancer[ J ]. N Engl JMed, 2013,369(12) : 1106-1114 .

5. Frias-Staheli N, Giannakopoulos N, Kikkert M, Taylor S, Bridgen A, et al. (2007) Ovarian tumor domain-containing viral proteases evade ubiquitin and ISG15-dependent innate immune responses[ J ]. Cell Host \& Microbe. pp 404-416.

6. M.E.Sowa, E.J.Bennett, S.P.Gygi, J.W.Harper. Defining the human deubiquitinating enzyme interaction landscape[ $\mathrm{J}]$. $\begin{array}{llll}\text { Cell } & 138 & \text { (2009) } & \text { : }\end{array}$ S.M. Nijman, M.P. Luna-Vargas, A. Velds, T.R. Brummelkamp, A.M. Dirac, T.K. Sixma, R. Bernards. A genomic and functional inventory of deubiquitinating enzymes[ J ]. Cell 123 (2005): 773-786.

7. Ernst R, Mueller B, Ploegh H, Schlieker. The Otubain YOD1 Is a Deubiquitinating Enzyme that Associates with p97 to Facilitate Protein Dislocation from the ER[ $\mathrm{J}$ ]. MOLECULAR CELL,2009:

$28-38$. Pickart CM, Cohen RE Proteasomes and their kin: proteases in the machine age[ J ]. Nat Rev Mol Cell Biol,2004,(5): 177187.

8. Sun X x, Challagundla K B , D aiM S. Positi ve reg u lation ofp53 stability and activi tyby the deubiquitinating enzym e otubain 1[ J ]. E M BO J, 20 12,31 (3 ): $57 \quad 6-92$ Herhaus L,A1 - Sa lihiM , M aeartney T, et a1. OTUB1 enhances TG F I 3 signalling by inhibiting the ubiquitylation and degradation of active SM D2/3 [ J ]. N at c ommun ,2013 
, $4: 2519$.

9. Messick TE, Russell NS, Iwata AJ, Sarachan KL, Shiekhattar $\mathrm{R}$, et al. Structural basis for ubiquitin recognition by the OTU1 ovarian tumor domain protein[ $\mathrm{J}$ ]. Journal of Biological Chemistry (2008) 283: 11038-11049.

10. Nijman SMB, Luna-Vargas MPA, Velds A, Brummelkamp TR, Dirac AMG, et al. A genomic and functional inventory of deubiquitinating enzymes[ J ]. Cell(2005)123: 773-786.

11. Makarova KS, Aravind L, Koonin EV A novel superfamily of predicted cysteine proteases from eukaryotes, viruses and Chlamydia pneumoniae[ $\mathrm{J}$ ].Trends in Biochemical Sciences (2000) 25: 50-52.

12. Amerik A, Hochstrasser M. Mechanism and function of deubiquitinating enzymes[ $\mathrm{J}$ ]. Biochimica Et Biophysica Acta-Molecular Cell Research. (2004)pp 189-207.

13. Inoue $\mathrm{Y}$, Miyamoto S, Fukami T, Shirota K, Yotsumoto F, Kawarabayashi T. Amphiregulin is much more abundantly expressed than transforming growth factor-alpha and epidermal growth factor in human follicular fluid obtained from patients undergoing in vitro fertilization-embryo transfer[ J ]. Fertil Steril 2009; 91:1035-1041.

14. Peluffo MC, Ting AY, Zamah AM, Conti M, Stouffer RL, Zelinski MB, Hennebold JD. Amphiregulin promotes the maturation of oocytes isolated from the small antral follicles of the rhesus macaque[ J ]. Hum Reprod 2012; 27:2430-2437.

15. Zhong J, Zhao M, Ma Y, et al. UCHL1 acts as a colorectal cancer oncogene via activation of the $\beta$-catenin / TCF pathway through its deubiquitinating activity[ J ]. Int J Mol Med, 2012, 30(2):430 -6.

16. Sun,X.X.,Challagundla,K.B., and Dai,M.S. Positive regulation of p53 stability and activity by the deubiquitinating enzyme Otubain 1[ J ]. EMBO J31;2012: 576-592 . 